\title{
Influenza Transmission in Preschools: Modulation by contact landscapes and interventions
}

\author{
A.A. Adalja ${ }^{1}$, P.S. Crooke ${ }^{2}$, and J.R. Hotchkiss ${ }^{3 *}$ \\ ${ }^{1}$ Center for Biosecurity and Department of Critical Care Medicine, University of Pittsburgh \\ ${ }^{2}$ Department of Mathematics, Vanderbilt University \\ ${ }^{3}$ Departments of Critical Care Medicine and Medicine, University of Pittsburgh
}

\begin{abstract}
Epidemiologic data suggest that schools and daycare facilities likely play a major role in the dissemination of influenza. Pathogen transmission within such small, inhomogenously mixed populations is difficult to model using traditional approaches. We developed simulation based mathematical tool to investigate the effects of social contact networks on pathogen dissemination in a setting analogous to a daycare center or grade school. Here we show that interventions that decrease mixing within child care facilities, including limiting the size of social clusters, reducing the contact frequency between social clusters, and eliminating large gatherings, could diminish pathogen dissemination. Moreover, these measures may amplify the effectiveness of vaccination or antiviral prophylaxis, even if the vaccine is not uniformly effective or antiviral compliance is incomplete. Similar considerations should apply to other small, imperfectly mixed populations, such as offices and schools.
\end{abstract}

Key words: influenza, Monte Carlo model, preschool, landscape ruggedness.

AMS subject classification: $92 \mathrm{C60}$.

\section{Introduction}

Epidemiologic data suggest that schools, daycare facilities, and other small, non-homogenously mixed venues likely play a major role in the dissemination of influenza. Schools and daycare centers have been labeled as virus exchange centers that can promote viral dissemination during

\footnotetext{
${ }^{*}$ Corresponding author. E-mail: hotchkissjr@upmc.edu
} 
epidemics [1]. Children can serve as super-spreaders of influenza due to prolonged viral shedding and higher quantities of shed virus [2]. Dissemination of pathogens within small populations is a random process and prone to high levels of variability, which can include spontaneous extinction (random "quenching") of an outbreak within a facility. In addition, most populations are not well mixed- not every individual interacts equally with all other individuals in the populationcreating barriers to transmission that are difficult to address with traditional, differential equation based modeling approaches. Because the dynamics of interpersonal interactions within individual venues are not explicitly addressed, current models cannot predict the changes in pathogen acquisition risk that accompany modifications of the contact network within a given facility. Similarly, the potential for quenching of an outbreak within small venues, and the modulation of this likelihood by interventions such as vaccination or antiviral therapy, remain occult. Individual based models (IBMs) can explicitly address contact patterns within small populations. Such contact models can be coupled with an appropriate transmission model that accounts for the random nature of transmission events (not every contact results in transmission), variation of infectiousness over time, and differences in susceptibility due to acquired immunity or antiviral treatment. Such integrated IBMs can be cast in a simulation framework in which the locations of the initially infectious individuals are randomly assigned, and the average dynamics of an outbreak studied over multiple simulations. Recent investigations have employed individual-based models to predict effects of different pathogen containment strategies on the spread of highly virulent influenza through large populations- cities, nations, and geographic regions $[3,4,5,6,7]$. In these models, the risk of infection faced by an individual as he or she visits a particular facility is assigned based on pathogen prevalence, pathogen transmissibility, and demographic aspects of the venue. Such phenomenological approaches cannot predict the consequences of deliberate changes in the contact patterns between individuals within the facility $[8,9,10,11]$. We developed an individual based simulation tool to investigate the effects of spatial heterogeneity on pathogen dissemination in a setting analogous to a daycare center or grade school. We use this tool to investigate the modulation of dissemination by within-facility social contact patterns, as well as the putative effects of feasible pharmacologic and vaccine based interventions.

\section{Model and Parameters}

The model comprises a population of 24 children followed over thirty days, comprising four 5-day school weeks with 4 interspersed weekends. Children are in the facility for 8 hours per day during school weeks, and have no contact with each other on weekends. The infectiousness of children continues to evolve (their clock "keeps running") during weekends, but they have no contact with each other. While in the facility, children associate in primary social contact clusters (such as tables or small social groups) ranging in size from 2 children to 12 children $(2,3,4,6$, 8, and 12 children per cluster); a cluster size of 24 children was also evaluated, corresponding to the traditional assumption of "uniform mixing." The total number of contacts of sufficient intensity to allow transmission between an infectious and an uninfected child in a 15 minute period was set at 2 


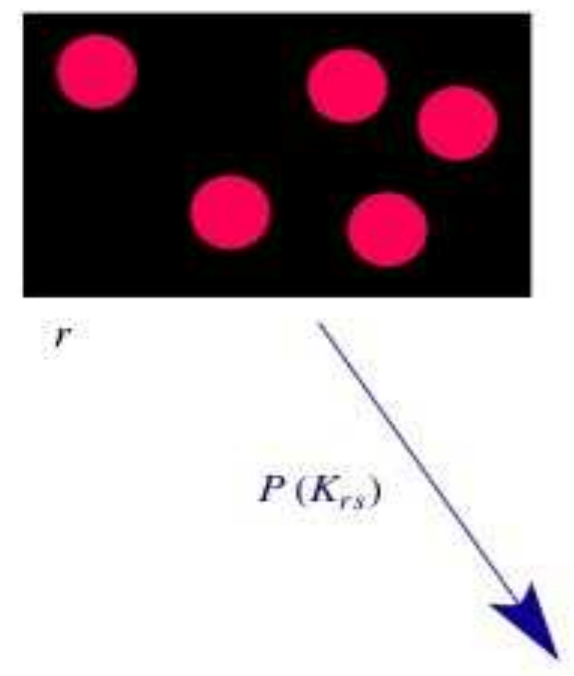

$$
P\left(K_{i j}\right)=\text { probability of contact between clusters }
$$
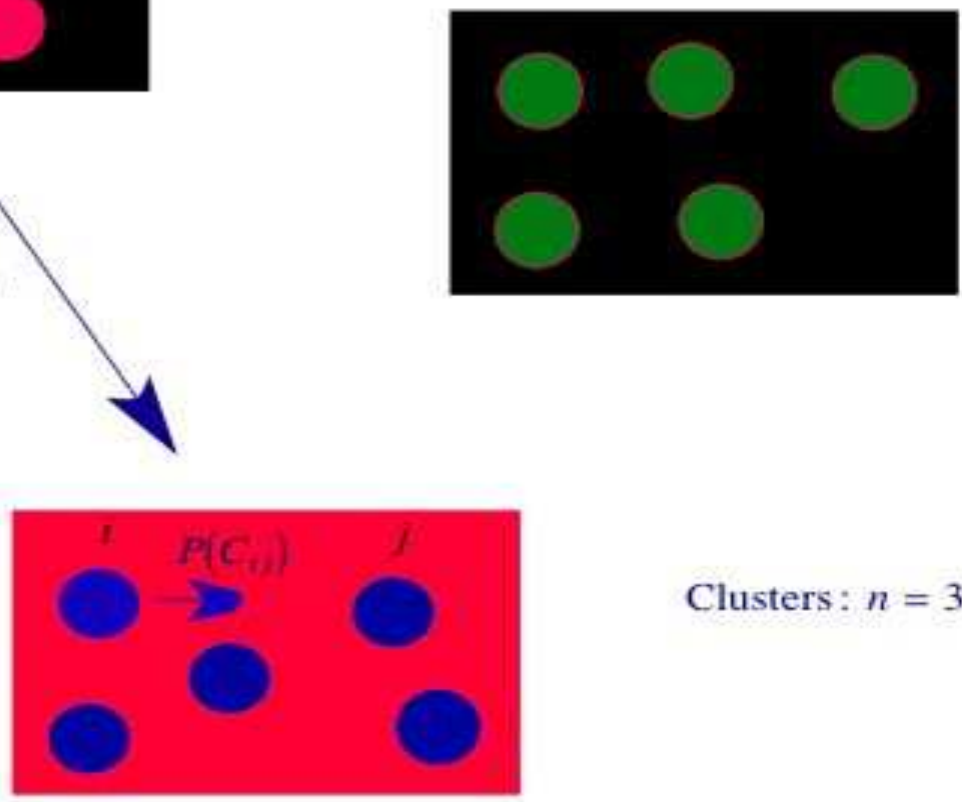

$s$

$$
P\left(C_{i j}\right)=\text { probability of contact within cluster }
$$

Figure 1: Schematic depiction of within- and extra- cluster contacts for 3 clusters each containing 3 children.

for each child. The number of contacts with children outside the childs primary cluster was varied from 0 (only contact within the cluster at a rate of 8 per hour) to 1 ( 4 within cluster and 4 outside cluster contacts each hour). The pattern of social contacts (potentially infectious encounters) was thus one of frequent encounters with members of an immediate social cluster interspersed with potentially less frequent encounters with individuals from the larger population of children (Figure 1). The resultant plots of the number of potentially infection-transmitting contacts between each pair of children over the course of a day (the "contact landscape") are characterized by "peaks and plains," with the relative height of the peaks determined by the probability of within-and withoutcluster contacts (figure 2).

The range of peak infectiousness examined spanned a per-encounter risk of transmission ranging from $0.4 \%$ to $1.8 \%$. The temporal pattern of infectiousness was adapted from Burke et al. [4]. Each simulation was seeded with a single infected (for $16 h$ ) child in a random position, emulating a child who acquired the infection $e . g$., on a school bus whilst returning home the previous day. 

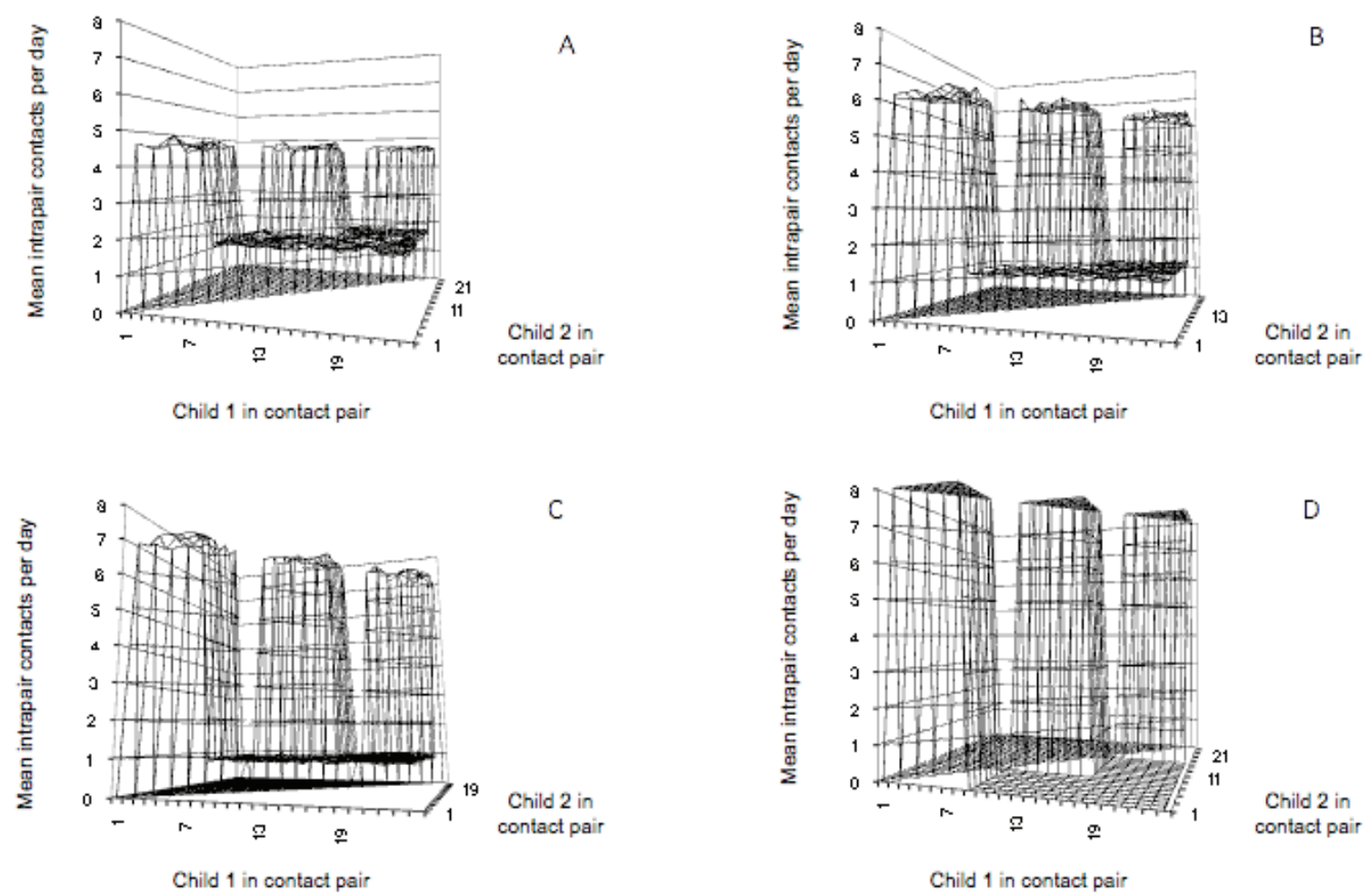

Figure 2: Contact landscape over one day with 8 children per cluster and probability of extracluster contact that is equal to (panel A), one-half (panel B), one third (panel $\mathbf{C}$ ) of the probability of contact within cluster. Panel D: contact landscape with no extracluster contacts.

Beyond these constraints, the contact patterns and infectious transmissions were both fully random. We explored 4 scenarios. In the first, there was no intervention. In the second, $50 \%$ of the simulated children were selected at random and treated with an antiviral agent that reduced their susceptibility $60 \%$ and that reduced the infectiousness of an infected child by $62 \%[7,12,13,14]$. In the third, all simulated children were treated with a vaccine that rendered $33 \%$ of the children immune (susceptibility zero, infectiousness zero). In the fourth, all children were treated with a vaccine that rendered $66 \%$ of the children immune. These very conservative scenarios were selected to highlight the interactions between imperfect or non-universally applied interventions and the characteristics of pathogen transmission in small and partially mixed populations. The ruggedness of the contact landscape for each combination of cluster size and density of extracluster contacts was defined as the square root of the squared sums of the average number of contacts between each pair of children each day. This measure integrates both the intensity and the breadth of contacts within the population, accounting for contacts within the childs primary social cluster and the (potentially less frequent) contacts with the larger population of children within the facility.

The model is comprised of 3 elements: the first two being an infectiousness, susceptibility and transmission model and a contact network model. The former describes the evolution (exposed $\rightsquigarrow$ infectious $\rightsquigarrow$ immune and noninfectious) of children afflicted with the pathogen, while the latter 
describes the patterns of child-child contact within the facility. The infectiousness/contact network model is embedded in a Monte Carlo simulation framework.

The pattern of infectiousness of a child was modeled as following a biexponential pattern consistent with the published data of Burke et al. (Figure 1, [4]). Briefly, following an exposure resulting in infection, there is a latent period during which the child is non-infectious, followed by a rise in infectiousness to a peak value, with a subsequent decline to zero. Once infected, children cannot be re-infected. The value of infectiousness is defined as the probability that a contact between a fully infectious child and a fully susceptible child will result in transmission of the virus to the susceptible child. The susceptibility of a child is defined as the probability that, on fully effective contact with a maximally infectious child, the uninfected child will become infected. For each potential transmission event (infected child encounters uninfected child), the probability of the uninfected child becoming infected is calculated as the product of the infectious childs infectiousness and the uninfected childs susceptibility. In the calculations presented, pharmacologic treatment is assumed to reduce susceptibility by $60 \%$ and infectiousness by $62 \%$.

\section{Computational Implementation and Results}

One hundred trials of each combination of cluster size, probability of extracluster contacts, and intervention were run for each of 16 putative transmissibilities. Each trial, encompassing 24 children, ran for 30 simulated days. Except where noted, the data presented for $R_{0}$, quenching, and landscape ruggedness are average values over the 100 trials at each of 16 levels of transmissibility. The software is written to run in Microsoft Excel. The software and the raw datasets can be obtained by interested investigators, free of charge, from the authors.

\subsection{Infectiousness/Susceptibility/Transmission Model}

When evaluated across all values of transmissibility and cluster size, and for all densities of extracluster contact is greater than zero, the observed values for the reproductive number $\left(R_{0}\right)$ in the absence of any intervention were a linearly declining function of landscape ruggedness. In the special case of no extracluster contacts, the relationship was exponentially declining (Figure $3)$. These effects were modulated by pathogen transmissibility, and were also observed in the settings of antiviral administration and vaccination. In contrast, the likelihood of quenching (mean $R_{0}<1$ over multiple realizations for injection of a single infected child) was primarily a function of transmissibility.

Decreasing the size of social contact clusters, or diminishing the frequency of contact between children residing in different clusters, lowered the value of $R_{0}$ even in the absence of any other intervention (Figure 4). These effects were most dramatic at cluster sizes of less than 12 children per cluster. Antiviral treatment or vaccination greatly reduced observed Ro and attenuated the differences due to cluster size. The probability of quenching following introduction of a single infectious child was minimally dependent on between- cluster mixing, and only displayed significant modulation by cluster size as cluster fell below 4 (Figure 5). 


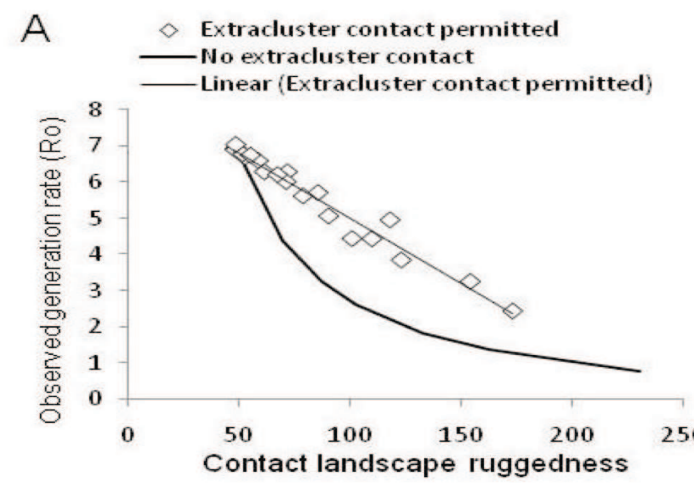

$\mathrm{B}$
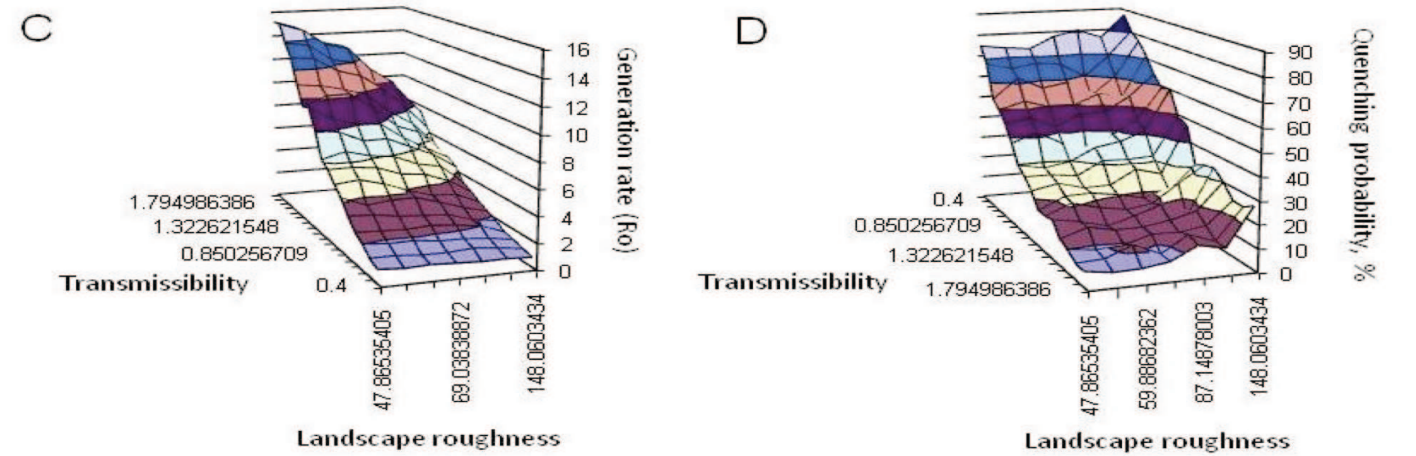

Figure 3: Effects of landscape ruggedness on observed $R_{0}$ and probability of quenching in the absence of pharmacologic intervention or vaccination (base case scenario). Panel A: Modulation of observed $R_{0}$ by contact landscape ruggedness over cluster sizes ranging from 2 to 24 children, with probability of extracluster contact equal to $0,1,1 / 2$, and $1 / 3$ that of intracluster contact over full ranges of transmissibility and cluster size. Panel B: Percent of realizations anticipated to spontaneously quench $\left(R_{0}<1\right)$ as a function of transmissibility over all levels of landscape ruggedness. Panel C: Observed $R_{0}$ as a function of landscape ruggedness and transmissibility over all cluster sizes. Panel D: Probability of quenching as a function of transmissibility and landscape ruggedness over all cluster sizes. 

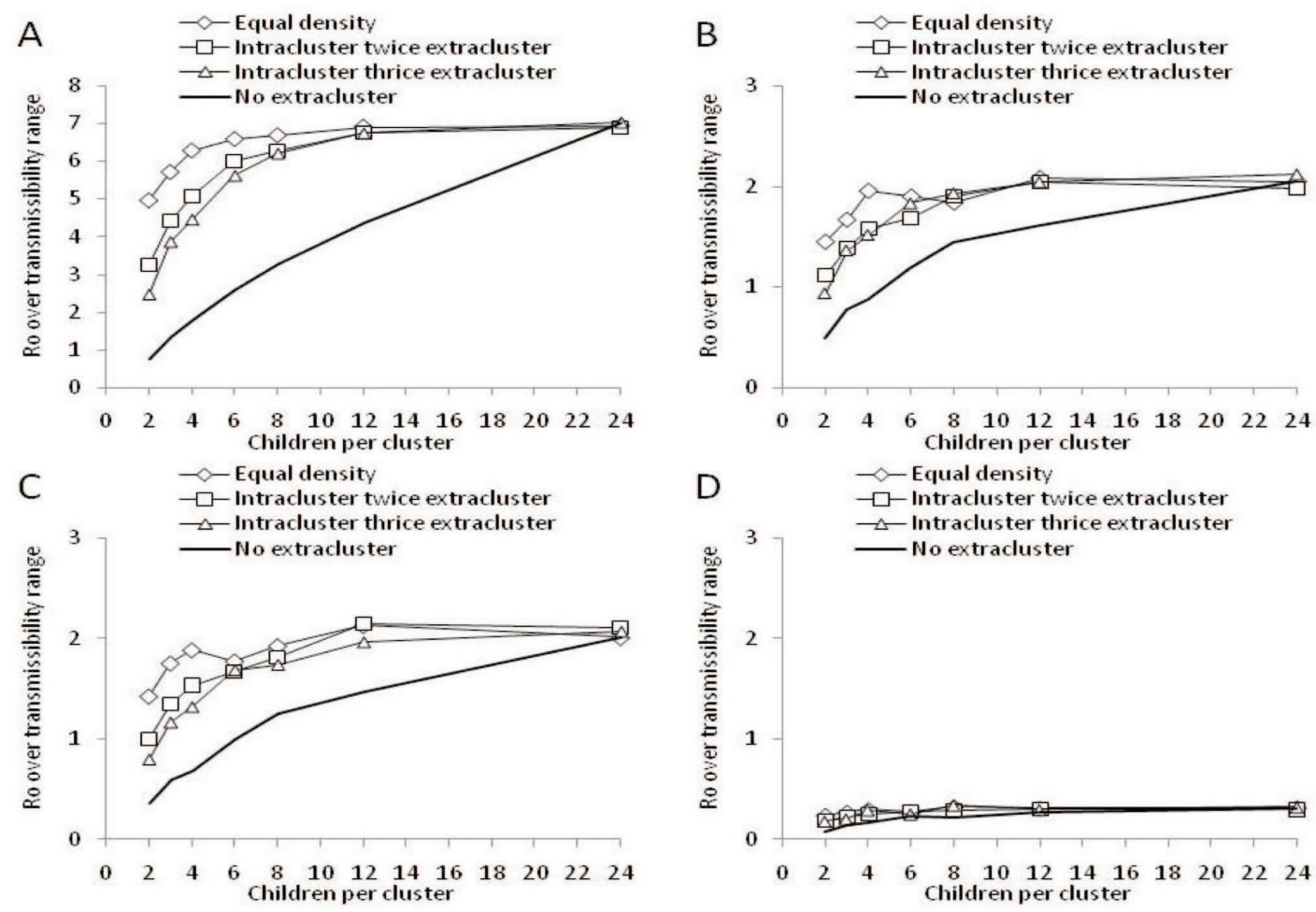

Figure 4: Values for $R_{0}$ with one initially infected child as a function of cluster size, degree of mixing between clusters, and adjuvant interventions. Panel A: Mean $R_{0}$ across all transmissibilities as a function of social cluster size and probability of encounters with children outside of the primary social cluster. Panel B: as for panel A, except with treatment of $50 \%$ of children with an antiviral agent that reduces susceptibility by $60 \%$ and infectiousness $62 \%$. Panel C: as for Panel A except with treatment of all children with a vaccine that abrogates susceptibility and infectiousness in 33\% of treated children. Panel D: as for Panel A except with treatment of all children with a vaccine that abrogates susceptibility and infectiousness in $66 \%$ of treated children. Note change of scale between Panel A and Panels B-D. 


\subsection{Contact Network Model}

Each day is partitioned into 96 fifteen minute intervals, and contacts between children are analyzed on this discrete temporal grid. Time in school (set at $8 h$ ) and time at home $(16 h)$ are treated separately. Children are in the facility for 5 days per week and have no contact with each other on weekends. The population of children is partitioned into distinct subgroups, or clusters, of equal sizes; cluster size is determined by the number of clusters and the total number of children. The probability of contact between each pair of children during an interval is specified. The probability of contact with another child in the primary cluster is set to attain the desired time density of intracluster contacts. In the setting of cluster size 2 , the within cluster contact density was set at the desired level $(\mathrm{N})$ and the likelihood of transmission was treated as $1-P$ (no. transmission in $N$ contacts)]. Similarly, the probability of contact with any child outside the primary cluster is set to attain the specified density of extracluster contacts given the number of children outside the primary cluster. For clarity, except for the initial seeding, no infections were assumed to arise at home. The infectiousness time course of children afflicted at school continued to evolve while they were at home (the clock continued running). Similarly, children did not contact each other during the weekend, but the infectiousness time continued to be incremented. Antiviral therapy was assumed to reduce susceptibility by $60 \%$ and infectiousness by $62 \%$, and-where applicable-was modeled as successfully implemented in $50 \%$ of the daycare population. Vaccination was assumed to render $33 \%$ or $66 \%$ of children completely immune and non-infectious.

Landscape ruggedness was explicitly computed for each simulation as

$$
R=\sqrt{\sum_{i, j=1}^{n}\left(\frac{C_{i j}}{N_{\text {real }} N_{\text {day }}}\right)^{2}}
$$

where $C_{i j}$ is the number of contacts between child $i$ and child $j$.

\section{Discussion and Conclusion}

"Fracturing" of the contact landscape, as by disparate social contact groups, dramatically reduces pathogen dissemination in small, partially structured populations. The extent of such fracturing can be captured by a composite index- contact landscape ruggedness-that accounts for both higher density of contacts within primary social groups and the degree of mixing between children in different groups. Nonuniform mixing patterns, together with the probabilistic character of transmission, render even incomplete population level interventions more effective in small groups such as those we examine. These findings are robust at levels of transmissibility that far exceed those anticipated for pandemic influenza, as the maximal observed value for $R_{0} \approx 14$ and the mean over all ensembles was approximately 5 (data not shown). This large range was deliberately chosen to ensure that the findings regarding social distancing are not dependent on limited transmissibility. 

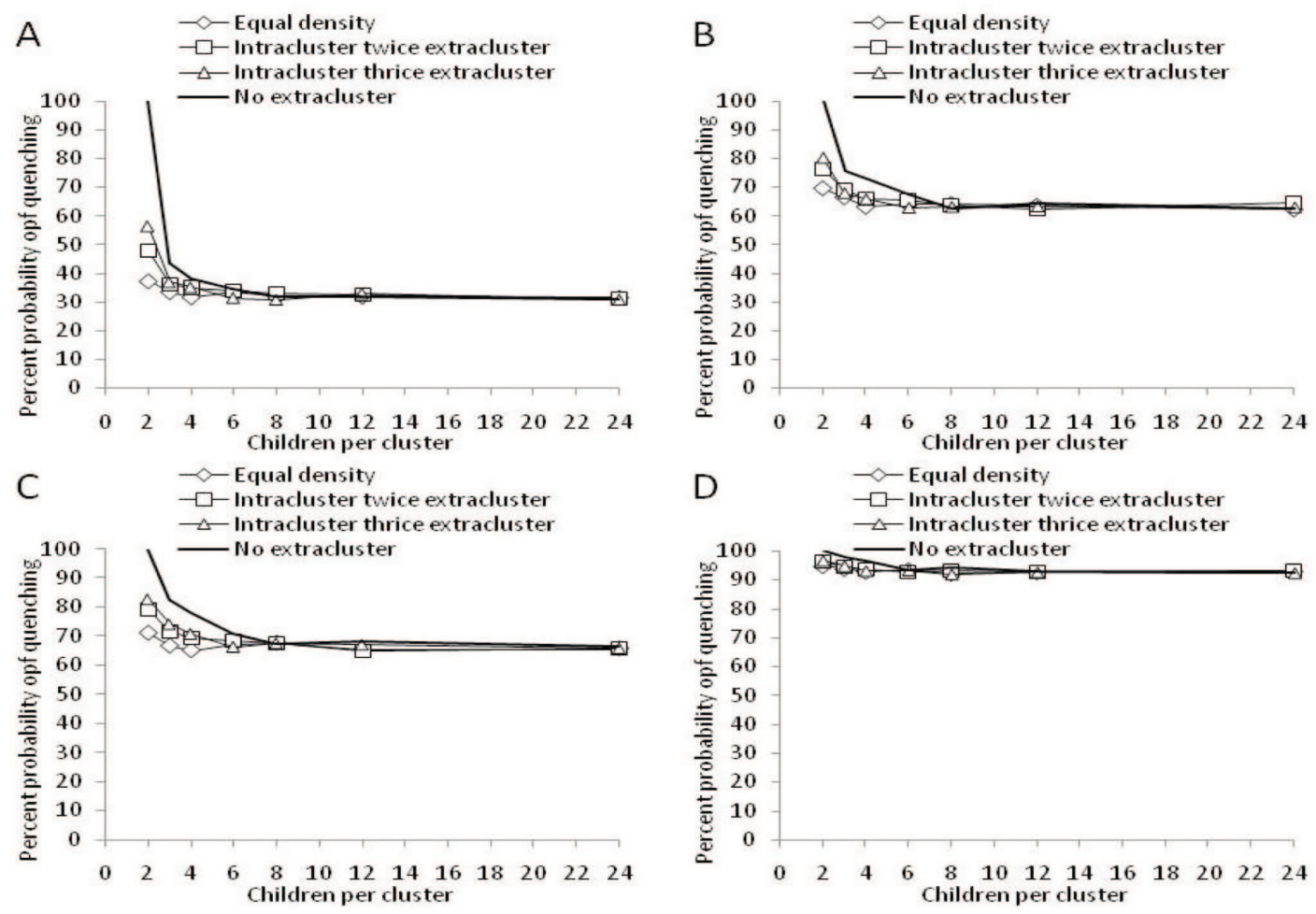

Figure 5: : Number of realizations that quench $\left(R_{0}<1\right)$ with one initially infected child as a function of cluster size, degree of mixing between clusters, and adjuvant interventions. Panel A: Mean $R_{0}$ across all transmissibilities as a function of social cluster size and probability of encounters with children outside of the primary social cluster. Panel B: as for Panel A, except with treatment of $50 \%$ of children with an antiviral agent that reduces susceptibility by $60 \%$ and infectiousness $62 \%$. Panel $\mathbf{C}$ : as for Panel A except with treatment of all children with a vaccine that abrogates susceptibility and infectiousness in 33\% of treated children. Panel D: as for Panel A except with treatment of all children with a vaccine that abrogates susceptibility and infectiousness in $66 \%$ of treated children. 
These predictions extend those of other investigators, who have found that social distancing interventions might prove useful in the setting of a pandemic. In fact, data from the 1918 influenza outbreak have shown that cities in which social distancing was practiced (in the form of school closures and public gathering bans) suffered less from the pandemic [15]. In the much smaller population we modeled, stochastic variation can lead to spontaneous quenching of an outbreak. Interventions that shift the dissemination balance even modestly-such as partial coverage with an antiviral agent or an imperfect vaccine-can couple with a fractured contact landscape to increase the likelihood of spontaneous viral extinction, rendering small facilities more resistant to incursion and less effective generators of new cases.

Such considerations could have straightforward public health implications. Specifically, reducing the size of contact groups and limiting contact between children in different groups are feasible interventions of low cost. While not predicted to have the same magnitude of effect as antiviral coverage or vaccination, these interventions could be implemented on a near-universal basis and would be anticipated to reduce the number of infectious children returned to the community, as well as augmenting the effectiveness of administration of antiviral agents or vaccines.

It is likely that the epidemiologic "signal" associated with the early incursion of pandemic influenza within a specific geographic region will be somewhat temporally diffuse [16]. This reality mitigates against "all or nothing," large scale social distancing or pharmacologic responses, as closing schools in response to any rise in febrile illnesses would be highly disruptive and would deplete, potentially to no benefit, limited stockpiles of antiviral agents. Also of note, the effectiveness of within facility spatial segregation (assigning children deemed at high risk of being infectious to separate "high risk" rooms while keeping schools open) would likely be highly dependent on the performance characteristics of the screening instrument used for this assignment. Some infectious children could still be left within "low risk" rooms, whilst some uninfected children could be exposed to elevated risk by being assigned to the "high risk" rooms. ([17]; Figures 4 and 5 of [18])

Within-facility social distancing (such as working with smaller clusters of children within the classroom, segregating such clusters to different parts of the room, and avoiding periods of more uniform between cluster mixing- such as lunch breaks or gym classes) could be a useful and only modestly disruptive early response. This strategy could be readily implemented in parallel with a high risk room spatial segregation policy (by restricting contact patterns in all rooms), in which case it would diminish the risks attending misclassification.

The implications of focusing pandemic preparedness on children has been described in other simulation models where immunizing only $20 \%$ of children was more effective than immunizing $90 \%$ of the elderly and a $70 \%$ immunization rate may protect the entire community $[19,20]$. Our results add support to the contention that vaccinating children (and others who cohabitate in contact intensive environments) should be a high priority, as the strong interactions predicted between landscape ruggedness, vaccination, and the generation rate of new infectious individuals suggests that this approach would provide a larger benefit than vaccinating those who do not routinely inhabit such contact intensive environments. 


\section{References}

[1] L.J. Radonovich, B.S. Bender. Children Should Be Among the Highest Priority Groups to Receive Immunization for Seasonal and Pandemic Influenza. Biosecurity and Bioterrorism: Biodefense Strategy, Practice, and Science, 5(2007), No. 4, 363-366.

[2] K.M. Neuzil, Y. Zhu, M.R. Griffin, K.M. Edwards, J.M. Thompson, S.J. Tollefson, P.F. Writght. Burden of interpandemic influenza in children younger than 5 years: A 25-year prospective study. J. Infect. Dis., 185(2002), No. 2, 147-152.

[3] I.M. Longini, A. Nizam, S.F. Xu, K. Ungchusak, W. Hanshaoworakul, D.A.T. Cummings, M.E. Halloran. Containing pandemic influenza at the source. Science, 309(2005), 10831087.

[4] N.M. Ferguson, D.A.T.. Cummings, S. Cauchemez, C. Fraser, S. Riley, A. Meeyai, S. Iamsirithaworn, D.S. Burke. Strategies for containing an emerging influenza pandemic in Southeast Asia. Nature, 437(2005), No. 7056, 209-214.

[5] T.C. Germann, K. Kadau, I.M. Longini, C.A. Macken. Mitigation strategies for pandemic influenza in the United States. Proc Natl. Acad. Sci. USA, 103(2006), No. 15, 5935-5940.

[6] N.M. Ferguson, D.A.T. Cummings, C. Fraser, J.C. Cajka, P.C. Cooley, D.S. Burke. Strategies for mitigating an influenza pandemic. Nature, 442(2006), No. 7101, 448-452.

[7] M.E. Halloran,N.M. Ferguson, S. Eubank, I.M. Longini, D.A.T. Cummings, B. Lewis, S.F. Xu, C. Fraser, A. Vullikanti, T.C. Germann, D. Wagner, R. Beckman, K. Kadau, C. Barrett, C.A. Macken, D.S. Burke, P.C. Cooley. Modeling targeted layered containment of an influenza pandemic in the United States. Proc. Natl. Acad. Sci. USA, 105(2008), No. 12, 4639-4644.

[8] L. Ancel Meyers, M.E. Newman, M. Martin, S. Schrag. Applying network theory to epidemics: control measures for Mycoplasma pneumoniae outbreaks. Emerg. Infect. Dis., 9(2003), No. 2, 204-210.

[9] S. Bansal, B.T. Grenfell, L.A. Meyers. When individual behaviour matters: homogeneous and network models in epidemiology. JR. Soc. Interface, 4(2007) No. 16, 879-891.

[10] J.R. Hotchkiss, D.G. Strike, D.A. Simonson, A.F. Broccard, P.S. Crooke. An agent-based and spatially explicit model of pathogen dissemination in the intensive care unit. Crit. Care. Med., 33(2005), No. 1, 168-176.

[11] W.G. Wilson. Resolving discrepancies between deterministic population models and individual-based simulations. Am. Nat., 151(1998), No. 2, 116-134.

[12] M.E. Halloran, F.G. Hayden, Y. Yang , I.M. Longini, A.S. Monto. Antiviral effects on influenza viral transmission and pathogenicity: observations from household-based trials. Am. J. Epidemiol., 165(2007), No. 2, 212-221. 
[13] F.G. Hayden, R.L. Atmar, M. Schilling,C. Johnson, D. Poretz, D. Paar, L. Huson, P. Ward, R.G. Mills. Use of the selective oral neuraminidase inhibitor oseltamivir to prevent influenza. N. Engl. J. Med., 341(1999), No. 18, 1336-1343.

[14] F.G. Hayden, J.J. Treanor, R.S. Fritz, M. Lobo, R.F. Miller, N. Kinnersley, R.G. Mills, P. Ward, S.E. Straus. Use of the oral neuraminidase inhibitor oseltamivir in experimental human influenza: randomized controlled trials for prevention and treatment. J. Am. Med. Assoc., 282(1999), No. 13, 1240-1246.

[15] D. Weycker, J. Edelsbeg, M.E. Halloran, I.M. Longini, A. Nizam, V. Ciuyla, G. Oster. Population-wide benefits of routine vaccination of children against influenza. Vaccine, 23(2005), No. 10, 1284-1293.

[16] M.M. Wagner, L.S. Gresham, V. Dato. Case. Detection, Outbreak Detection and Outbreak Characterization. M.M. Wagner, A.W. Moore and R.M. Aryel(Eds) In Handbook of Biosurveillance, Elsevier, Burlington, 2006, 27-51.

[17] J. Stein, J. Louie, S. Flanders, J. Maselli, J. Hacker, W.L. Drew, R. Gonzales. Performance characteristics of clinical diagnosis, a clinical decision rule, and a rapid influenza test in the detection of influenza infection in a community sample of adults. Ann. Emerg. Med., 46(2005), 412-419.

[18] J.R. Hotchkiss, P. Holley, P.S. Crooke. Analyzing Pathogen Transmission in the Dialysis Unit: Time for a (Schedule) Change? Clin. J. Am. Soc. Nephrol., 2(2007), No. 6, 1176-1185.

[19] I.M. Longini, M.E. Halloran, A. Nizam, M. Wolff, P.M. Mendelman, P.E. Fast, R.B. Belshe. Estimation of the efficacy of live, attenuated influenza vaccine from a two-year, multi-center vaccine trial: implications for influenza epidemic control. Vaccine, 18(2000), No. 18, 19021909.

[20] H. Markel, H.B. Lipman, J.A. Navarro, J.A. Navarro, A. Sloan, J. Michalsen, A.M. Stern, M.S. Cetron. Nonpharmaceutical interventions implemented by US cities during the 19181919 influenza pandemic. J. Am. Med. Assoc., 298(2007), No. 6, 644-654. 